\title{
Nonintegrable dynamics of the triplet-triplet spatiotemporal interaction
}

\author{
S. R. Lopes ${ }^{1}$ and F. B. Rizzato ${ }^{2, *}$ \\ ${ }^{1}$ Departamento de Física, Universidade Federal do Paraná, P.O. Box 19081, 81531-990 Curitiba, Paraná, Brazil \\ ${ }^{2}$ Instituto de Física, Universidade Federal do Rio Grande do Sul, P.O. Box 15051, 91501-970 Porto Alegre, \\ Rio Grande do Sul, Brazil \\ (Received 2 December 1998)
}

\begin{abstract}
In this paper we examine the coupling of two wave triplets sharing two common modes. The analysis is performed in the solitonic sector of the parameter space where uncoupled solutions departing from linearly unstable homogeneous initial conditions evolve into a collection of regularly interspersed, spatiotemporally localized spikes. The uncoupled system is integrable, but coupling destroys integrability. As coupling grows, energy transfer to smaller spatial scales does appear and becomes faster not only in linearly unstable, but also in linearly stable cases. Chaos in low-dimensional subsystems appears to be responsible for the transfer. We perform a series of numerical tests to verify this idea. [S1063-651X(99)08710-3]
\end{abstract}

PACS number(s): 05.45.Jn

\section{INTRODUCTION}

Resonant nonlinear three-wave coupling has an important role in both space and laboratory plasma processes, such as filamentation and anomalous absorption of laser beams [1], generation of auroral and solar radio emissions [2], and modulation of the solar wind Langmuir waves [3]. Given the interest on the subject, the conservative resonant triplet interaction has been fully investigated and shown to be completely integrable via inverse scattering [4]. Another related case of resonant interaction that has been attracting some renewed attention is the one involving two coupled triplets sharing two common modes [5-7] where, all in all, four independent waves undergo mutual interaction. This type of kinematics, where the interacting triplets share common modes, has been shown to be essential in nonlinear interactions involving electromagnetic, Langmuir, and ion-acoustic waves propagating in unmagnetized plasmas $[8,9]$, in magnetohydrodynamics interactions involving Alfvén and ionacoustic waves [10], and in plasma-beam interactions in the presence of negative energy waves [5].

These kinds of wave-wave couplings in continuous media are frequently described by amplitude equations. Once one identifies a set of high frequency modes interacting in the resonant fashion, one applies multiple scale formalism to derive equations governing the slow space-time evolution of these modes, the amplitude equations. Amplitude equations are justified when the spectral widths of both wave vector $(\mathbf{K})$ and frequency $(\Omega)$ spectral distributions of the interacting packets are respectively small as compared to the inverse spatial and temporal scale of the interaction $[11,12]$. It is the purpose of this paper to further analyze the amplitude dynamics of the coupled triplets.

Different from the temporal dynamics of the uncoupled triplets, the coupled temporal dynamics has been shown to produce amplitude equations allowing for temporal chaos [6]. To be more specific, if one removes all spatial depen-

\footnotetext{
*Fax: 55-51-3191762. Electronic address: rizzato@if.ufrgs.br
}

dence from the governing equations, then the solutions developing on this homogeneous manifold - sometimes we shall refer to the homogeneous manifold simply as HM can be chaotic if a number of conditions is fulfilled. Not much, however, has been said about solutions departing from the homogeneous manifold. One relevant question here would be on the stability of the homogeneous manifold against inhomogeneous perturbations. If, for instance, a certain homogeneous solution - not necessarily a static equilibrium - is perturbed by some small inhomogeneity, would this inhomogeneity grow in time? If the answer is positive we would have an effectively unstable homogeneous manifold. Otherwise, the manifold would be effectively stable. Previous findings show that HM's supporting chaotic orbits are always effectively unstable, while those supporting regular orbits may be effectively unstable against some types of perturbation and stable against others, all depending on the basic wave vector of the perturbing terms [7]. This latter kind of behavior, in particular, is what can be expected from conventional linear perturbative analysis, where by conventional we mean the linear stability analysis that assumes static backgrounds. Conventional stability is expected at this point because if the system is regular it spends so much time close to hyperbolic points that a static approximation becomes reasonable. On the other hand, what has been suggested to explain the instability of chaotic cases is that inhomogeneities tend to grow as a result of a stochastic pump provided by the chaotic orbits evolving on the HM [7]. The action of the stochastic pump would be similar to that of the Fermi-Pasta-Ulam problem, as analyzed recently [13]; the pump would act like a random source diffusively feeding energy into all the remaining modes of the system, and as a final result one would have the system moving towards some energy equipartitioned state. From the above comments, one sees that the pump model idea is general and thus expected to be applicable to a variety of settings. Indeed, we will suggest here that it also works well under more complicated conditions involving not only stable, but unstable HM's of the triplet-triplet interaction as well.

Given the above perspective, we have two basic goals 
with the present paper. One of them is to further investigate the ideas behind the stochastic pump model. To do that, we first focus our attention on settings displaying conventional linear stability. We make calculations assuming a static background, and simply choose wave vectors located outside the instability band. This sort of system shall be analyzed under two distinct conditions: when the HM is chaotic and when it is regular. We shall see that only when the homogeneous dynamics is chaotic, energy transfer takes place. Then we shall make tests on the stochastic pump model. We shall transform our homogeneous manifold into a real pump subsystem. In other words, we remove all the influence of spatially inhomogeneous modes onto the homogeneous manifold, maintaining the reverse influence - that of the homogeneous manifold on the remaining space-dependent modes. With this procedure we can actually look at the HM as a closed pump, feeding energy into all the remaining (space-dependent) modes, without loss of its own energy. As our numerical results show, there is agreement between this pump model and results arising from fully connected simulations, for short intervals of time; we call fully connected simulations those simulations which are completely selfconsistent and energy conserving. As time elapses, the HM of the fully connected simulations loses appreciable energy to the inhomogeneous modes. Given that in the pump model the HM is isolated and does not lose its energy, the two kinds of results should eventually diverge.

The other goal of the paper is to show that the idea of the pump model goes beyond the previous setting. We proceed as follows. The preceding analysis is extended into regimes where the uncoupled triplets display conventional linear instability leading to the formation of localized structures. Localization has not been appreciated before [7] because the appropriate basic condition $\mathrm{v}_{g_{2}}<\mathrm{v}_{g_{1}}<\mathrm{v}_{g_{3}}$ [4] had not been chosen; we denote by $\mathbf{v}_{g_{i}}, i=1,2,3,4$, the group velocities of the four relevant waves. In the uncoupled regime, condition $\mathbf{v}_{g_{2}}<\mathbf{v}_{g_{1}}<\mathbf{v}_{g_{3}}$ allows for soliton propagation and the existence of related localized structures. The idea here is to see what happens as coupling is turned on. There is a belief that in some settings solitons are so robust that, even in chaotic regimes, localized structures should be seen as asymptotic states of the dynamics [14]. We will see that this is not the case here. With coupling turned on, organized localized structures are rapidly destroyed with subsequent emission of short wavelength radiation. At this point we note that the transition to spatiotemporal chaos again depends on the existence of chaos in low-dimensional subsystems. Here, the HM alone is regular, otherwise one would not have solitons in the uncoupled limit $r \rightarrow 0$. Here the chaotic pump is formed not by the HM solely, but by the larger new subsystem composed by the HM along with the first spatial harmonics of the Fourier spectrum. As this new chaotic drive is connected to the rest, energy transfer takes place.

The plan of the paper goes as follows: in Sec. II we introduce the basic formalism, governing equations, and numerical methodology; in Sec. III we investigate the dynamics when inhomogeneous modes are all stable; and in Sec. IV we investigate when localized structures are formed as a result of linear instabilities of inhomogeneous modes. In Sec. V we conclude the work.

\section{MODEL}

In order to describe the mutual interaction of two wave triplets sharing two common modes and constituted, therefore, by four wave modes in all, we assume that the following resonant conditions are fulfilled:

$$
\begin{gathered}
\Omega_{\mathbf{K}_{1}} \approx \Omega_{\mathbf{K}_{3}}+\Omega_{\mathbf{K}_{2}}, \\
\mathbf{K}_{1}=\mathbf{K}_{2}+\mathbf{K}_{3}, \\
\Omega_{\mathbf{K}_{1}} \approx \Omega_{\mathbf{K}_{4}}-\Omega_{\mathbf{K}_{2}}, \\
\mathbf{K}_{1}=\mathbf{K}_{4}-\mathbf{K}_{2},
\end{gathered}
$$

where $\Omega_{\mathbf{K}_{j}}$ and $\mathbf{K}_{j},\{j=1,2,3,4\}$ are, respectively, the fast frequencies and fast wave vectors of the interacting waves; note that within the present formalism, the shared modes are modes 1 and 2 .

We allow for small frequency mismatches, a usual effect in wave-wave interaction. We shall see that the role of mismatches in the spatiotemporal triplet-triplet interaction is essential in determining the integrability properties of the wave dynamics. Frequency mismatch occurs because, even for perfectly matched wave vectors, the respective frequencies obtained from the linear dispersion relations may not quite be likewise matched. In laser accelerators and, in general, laser-plasma interactions, frequency mismatch can even enhance the linear growth rate in several situations $[8,15,16]$. Therefore, mismatched modes can be stronger and of greater importance in the dynamics. Wave vector mismatches could also be incorporated into the theory. However, as frequency and wave vector mismatches are formally equivalent, we focus attention on the former.

Following the model revisited in a series of recent papers [6-8], the dimensionless amplitude equations governing the one dimensional, spatiotemporal, slow modulational dynamics can be cast into the form

$$
\begin{aligned}
\frac{\partial A_{1}(x, t)}{\partial t}+\mathrm{v}_{g_{1}} \frac{\partial A_{1}(x, t)}{\partial x}= & A_{2}(x, t) A_{3}(x, t) \\
& -r A_{2}^{*}(x, t) A_{4}(x, t), \\
\frac{\partial A_{2}(x, t)}{\partial t}+\mathrm{v}_{g_{2}} \frac{\partial A_{2}(x, t)}{\partial x}= & -A_{1}(x, t) A_{3}^{*}(x, t) \\
& -r A_{1}^{*}(x, t) A_{4}(x, t),
\end{aligned}
$$

$\frac{\partial A_{3}(x, t)}{\partial t}+\mathrm{v}_{g_{3}} \frac{\partial A_{3}(x, t)}{\partial x}=i \delta_{3} A_{3}(x, t)-A_{1}(x, t) A_{2}^{*}(x, t)$,

$\frac{\partial A_{4}(x, t)}{\partial t}+\mathrm{v}_{g_{4}} \frac{\partial A_{4}(x, t)}{\partial x}=i \delta_{4} A_{4}(x, t)+r A_{1}(x, t) A_{2}(x, t)$.

$A_{j},\{j=1,2,3,4\}$, are the complex amplitudes of the four fields, $\delta_{3,4}=\Omega_{\mathbf{K}_{1}} \mp \Omega_{\mathbf{K}_{2}}-\Omega_{\mathbf{K}_{3,4}}$ are independent frequency 
mismatches corresponding to fields $A_{3}$ and $A_{4}$ (i.e., one can always take $\delta_{1}=\delta_{2}=0$ as we actually did), $r$ is a variable strength factor measuring the intensity of the triplet-triplet coupling [6-8], and $\mathrm{v}_{g_{j}}, j=1,2,3,4$, are the respective group velocities along the spatial modulation that we take as one dimensional (the $x$ axis) in this work. Time and space derivatives are first order as a result of our multiple time and space scales.

The set of governing equations (2)-(5) can be derived from a continuous Hamiltonian. Indeed, it is possible to see that the following relations hold

$$
\frac{\partial A_{j}(x, t)}{\partial t}=\frac{\delta H}{\delta A_{j}^{*}}, \quad \frac{\partial A_{j}(x, t)^{*}}{\partial t}=-\frac{\delta H}{\delta A_{j}},
$$

where we introduce the functional derivative

$$
\frac{\delta}{\delta A_{j}} \equiv \frac{\partial}{\partial A_{j}}-\frac{\partial}{\partial x} \frac{\partial}{\partial\left(\frac{\partial A_{j}}{\partial x}\right)}
$$

(the same definition holds if $A$ is replaced with $A^{*}$ ), and where the full Hamiltonian $\hat{H}$ is to be written in terms of the Hamiltonian density $H$ as

$$
\begin{aligned}
\hat{H} \equiv & \int d x H=\int d x\left[-A_{1} A_{2}^{*} A_{3}^{*}+A_{1}^{*} A_{2} A_{3}-r\left(A_{1}^{*} A_{2}^{*} A_{4}\right.\right. \\
& \left.\left.-A_{1} A_{2} A_{4}^{*}\right)+i \delta_{3}\left|A_{3}\right|^{2}+i \delta_{4}\left|A_{4}\right|^{2}-\sum_{j=1}^{4} \mathrm{v}_{g_{j}} A_{j}^{*} \frac{\partial A_{j}}{\partial x}\right] .
\end{aligned}
$$

The Hamiltonian does not depend explicitly on time; therefore, it is a time conserved quantity. In addition to the Hamiltonian, the following quantities are also conserved:

$$
\begin{aligned}
& C_{1}=\int d x\left[\left|A_{2}\right|^{2}-\left|A_{3}\right|^{2}+\left|A_{4}\right|^{2}\right], \\
& C_{2}=\int d x\left[\left|A_{1}\right|^{2}+\left|A_{3}\right|^{2}+\left|A_{4}\right|^{2}\right] .
\end{aligned}
$$

The arrangement of signs in $C_{1}$ and $C_{2}$ indicates that we can look at the whole process as a decay interaction with $A_{1}$ as the decaying pump; from this perspective, $A_{2}$ is an idler wave, whereas $A_{3}$ is a Stokes mode and $A_{4}$ an anti-Stokes mode. In the case of nonlinear interactions in unmagnetized plasmas for instance, $A_{1}$ is a transverse electromagnetic wave, $A_{2}$ is an ion-acoustic wave, and $A_{3}$ and $A_{4}$ are, respectively, Stokes and anti-Stokes Langmuir modes [8,9].

All those quantities will be useful in checking out the accuracy of our integration methods, which we outline now. The basic integration method is pseudospectral and the four fields $A_{j},\{j=1,2,3,4\}$, are Fourier analyzed according to

$$
A_{j}=\sum_{n=-N / 2+1}^{N / 2} a_{j_{n}}(t) e^{i n k x} .
$$

For further purposes, note that while $j$ denotes the wave type, $n$ denotes the mode number or harmonic number. The harmonic or modal amplitudes $a_{j_{n}}$ are integrated in time with a predictor-corrector algorithm. We use $N=64-256$ modes, removing half of them to cure aliasing. We denote the basic slow wave vector by $k$ and point out that, due to the structure of the equations, variations in $k$ can be absorbed in variations of the group velocities or vice-versa. Fluctuations of the conserved quantities, including energy, are not larger than one part in $10^{5}$, and variations of the tolerance factors of the integrator do not alter the final outcome of the runs. Our conclusions are insensitive on the values of $N$ used in the simulations.

\section{STABLE HOMOGENEOUS MANIFOLDS}

\section{A. Initial settings}

All full spatiotemporal simulations in this paper start out from initial conditions representing a static background pump

$$
\begin{gathered}
A_{1}(t=0)=a_{1_{o}}(t=0) \equiv \mathcal{A} \neq 0 \\
\text { and } \quad a_{j_{n}}(t=0)=0, \quad\{j=2,3,4\}, \quad \forall n,
\end{gathered}
$$

perturbed by small terms $A_{j}=a_{j_{1}} e^{i \kappa x}+a_{j_{-1}} e^{-i \kappa x}, a_{j_{ \pm 1}}$ $\sim e^{-i \omega t}$, such that $a_{j_{ \pm 1}} \ll|\mathcal{A}|$; this is the convenient choice to investigate the stability of a strong $A_{1}$ pump. We take

$$
\delta_{4}=0
$$

so a dispersion relation $\omega=\omega(\kappa)$ can be obtained from

$$
P_{3} P_{2}-P_{R}=0 \text {, }
$$

where

$$
\begin{gathered}
P_{2}=i\left(-\omega+\mathrm{v}_{g_{2}} \kappa\right), \\
P_{3}=i\left(-\omega+\mathrm{v}_{g_{3}} \kappa-\delta_{3}\right), \\
P_{4}=i\left(-\omega+\mathrm{v}_{g_{4}} \kappa-\delta_{4}\right),
\end{gathered}
$$

and

$$
P_{R}=|\mathcal{A}|^{2}\left(1-r^{2} \frac{P_{3}}{P_{4}}\right) \text {. }
$$

We point out that the Greek character $\kappa$ symbolizes the continuous wave vector of the dispersion relation, while $k$ denotes the basic wave vector to be used in the simulations. The dispersion relation (15) can be analyzed numerically, but we first briefly discuss some of its relevant results. For a given $\mathcal{A}$ and $\kappa$ positive, there exists an instability (complex $\omega$ 's) band extending from $\kappa=0$ up to a certain $\kappa_{\max }$. The larger the value of $\mathcal{A}$, the larger $\kappa_{\max }$. In general, maximum growth rates occur for $\kappa=0$, which contrast with several 

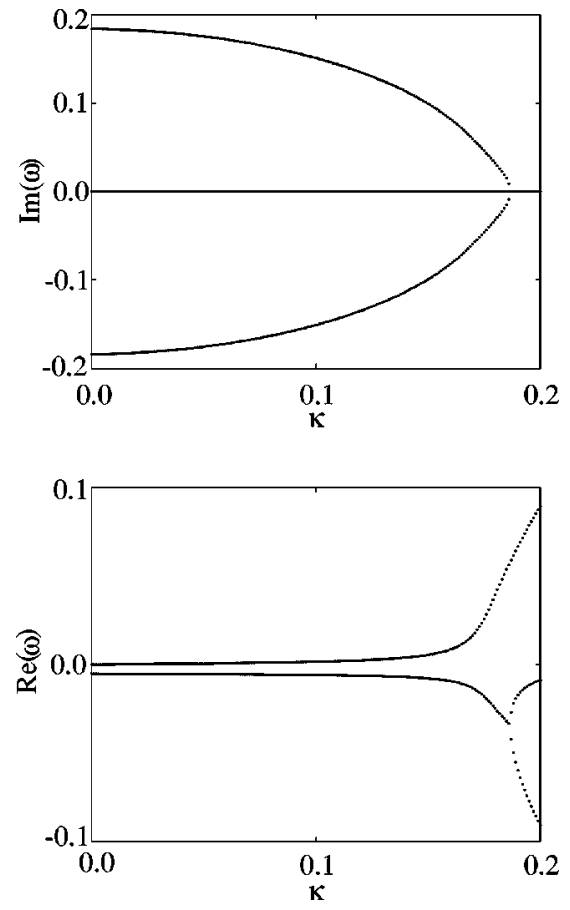

FIG. 1. Plot of the dispersion relation, Eq. (15) of the text $\mathcal{A}$ $=(0 ; 0.185), \delta_{3}=0.01, r=0.1, \mathrm{v}_{g_{1}}=0, \mathrm{v}_{g_{2}}=1$, and $\mathrm{v}_{g_{3}}=-0.5$.

other cases where the growth rate vanishes at $\kappa=0[14,17]$. The instability at $\kappa=0$ is precisely the one giving rise to the homogeneous dynamics $[6,8]$. Given that homogeneous perturbations are always unstable, if an inhomogeneous mode with $\kappa \neq 0$ falls within the instability band, one has a competing process involving the unstable dynamics on the HM $(\kappa=0)$ and the unstable dynamics off the HM $(\kappa=k \neq 0)$. We refer to Fig. 1 where we plot $\omega=\omega(\kappa)$ as calculated from Eq. (15) for $\mathcal{A}=(0,0.185), \delta_{3}=0.01$, and $r=0.1$. All of the mentioned properties can be seen there; in addition, note that one has two roots that fuse into one in unstable regions; the other root is always stable. The related problem of saturation of the instability is certainly far from trivial and we focus our attention on some particular cases.

\section{B. Conventionally stable homogeneous manifold}

The first case of interest is the one where no inhomogeneous mode $(k \neq 0)$ is unstable in the conventional sense. Will the instability and the ensuing dynamics on the HM induce some new type of instability that could cause growth of inhomogeneous perturbations? We recall that if the HM were completely stationary, there would be no chance for such a process to occur, since in this case the dispersion relation (15) would apply fully. The problem however, as said before, is that the HM cannot be seen as a totally stationary structure. In view of the existence of a $\kappa=0$ instability, some kind of dynamics, regular or chaotic, is bound to happen there. And this can alter the global stability properties of the system. From this point of view we shall analyze the dynamics in two distinct cases: regular and chaotic HM's. Again, as mentioned earlier, results from conventional linear stability calculations are expected to apply in the regular case. The fact is that if the orbits are regular, they spend so much time close to hyperbolic points that static approximations are reasonable. But the matter is more involved when chaos sets in as we shall examine next.

That the HM can be either regular or chaotic can be seen from the following initial reasoning and simulations. It has been shown [6] that the HM by itself is a two-degrees-offreedom (2DOF) conservative dynamical system. After some analytical work one can actually derive a governing Hamiltonian. To do so, from Eq. (11) we write the corresponding homogeneous fields in the form

$$
A_{j}(x, t) \equiv a_{j_{o}}(t),
$$

which does not depend on the spatial coordinate. Then we introduce real amplitudes and phases through

$$
a_{j_{o}}=\sqrt{\rho_{j}} e^{i \phi_{j}, \quad\{j=1,2,3,4\}}
$$

to obtain canonical conjugate equations for these quantities in the forms $\dot{\rho}=-\partial h / \partial \phi$ and $\dot{\phi}=\partial h / \partial \rho$, with a reduced governing Hamiltonian $h$ given in the form

$$
\begin{aligned}
h= & 2 \sqrt{\rho_{1} \rho_{2} \rho_{3}} \sin \left(\phi_{1}-\phi_{2}-\phi_{3}\right) \\
& -2 r \sqrt{\rho_{1} \rho_{2} \rho_{4}} \sin \left(\phi_{1}+\phi_{2}-\phi_{4}\right)-\delta_{3} \rho_{3}-\delta_{4} \rho_{4} .
\end{aligned}
$$

We now introduce new canonical variables $\phi_{1}-\phi_{2}-\phi_{3}$ $\rightarrow \phi_{1}, \quad \phi_{4}-2 \phi_{2}-\phi_{3} \rightarrow \phi_{4}, \quad \rho_{2} \rightarrow \rho_{2}-\rho_{1}-2 \rho_{4}, \quad \rho_{3} \rightarrow \rho_{3}$ $-\rho_{1}-\rho_{4}$, with the remaining variables unchanged, to turn Hamiltonian (18) into an explicit 2DOF one. Then, one makes Poincaré plots, recording the pair $\rho_{o}, \phi_{o}$ each time $d \rho_{4} / d t=0$, with $d^{2} \rho_{4} / d t^{2}<0$. One launches about 20 initial conditions whose energy is compatible with our standard initial condition given in Eq. (13), with $\mathcal{A}=(0,0.185)$. The results can be appreciated in Fig. 2. In panel (a) we use $r$ $=1.0$ and $\delta_{3}=0.0001$. The system is predominantly regular, with large dominance of uncorrupted Kolmogorov-ArnoldMoser $(K A M)$ [18] curves. This can be expected from results derived in a previous paper [19], where the author shows that the homogeneous dynamics at $\delta_{3}=0$ is exactly integrable, irrespective of $r$. Now one moves to panel (b) where $\delta_{3}$ $=0.1$ is used. For this larger mismatch, chaos is spread over most of the phase space. For a fixed $\delta_{3} \neq 0$, increasing values of $r$ also enhance chaotic activity.

In general, one thus sees that the HM can indeed support a nontrivial dynamics, either chaotic or regular. If the state of the homogeneous manifold were a static background, results of the dispersion relation (15) would apply. However, given the highly nontrivial dynamics developing on the manifold, the space time dynamics should be examined with care.

In order to monitor instabilities and energy transfer to inhomogeneous modes, we make use of a spectral average $[20,21]$, which enables one to estimate the number of active modes in the system. We denote this quantity by $\sqrt{\left\langle N^{2}\right\rangle}$ and define it as 
(a)

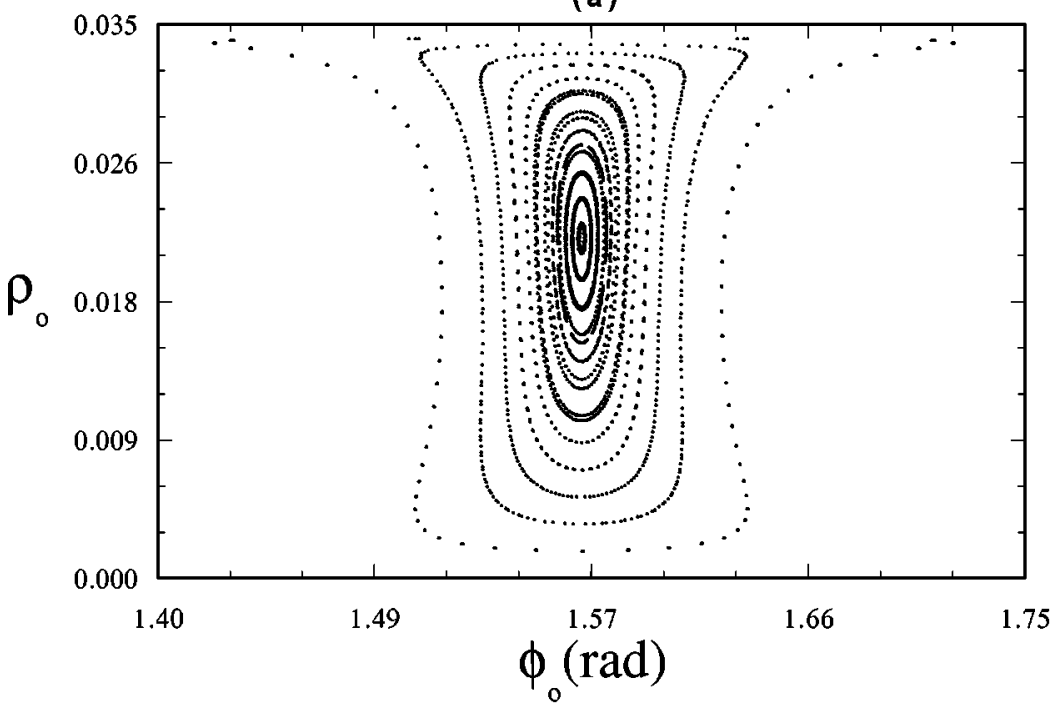

(b)

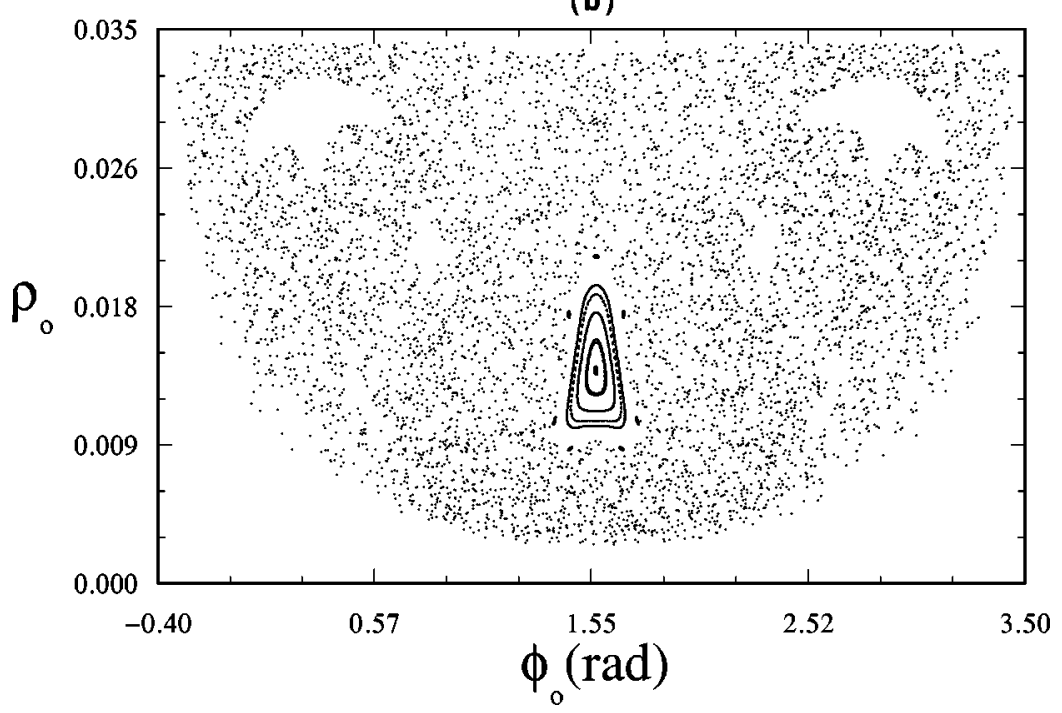

FIG. 2. Poincaré plots on the HM. A series of isoenergetic trajectories are plotted for the conditions discussed in the text: (a) $\delta_{3}=0.0001$ and (b) $\delta_{3}=0.1$. In both cases, $\mathcal{A}=(0,0.185)$ and $r=1$.

$$
\sqrt{\left\langle N^{2}\right\rangle}=\sqrt{\frac{\sum_{n=1}^{N} \sum_{j=1}^{4} n^{2}\left|a_{j_{n}}\right|^{2}}{\sum_{n=1}^{N} \sum_{j=1}^{4}\left|a_{j_{n}}\right|^{2}}} .
$$

From its definition one sees that $\sqrt{\left\langle N^{2}\right\rangle}$ is the square root of the averaged $n^{2}$, with the average weighted by the square of the mode number and type amplitudes. One could also further average over several similar initial conditions, but our results on energy transfer remain the same as long as the initial conditions are all simultaneously stable or all simultaneously unstable. $\sqrt{\left\langle N^{2}\right\rangle}$ is expected to grow in time in diffusive cases where more and more modes become involved in the dynamics. In the absence of energy transfer, $\sqrt{\left\langle N^{2}\right\rangle}$ remains limited by the number of linearly unstable modes; in the case of stable modes only, $\sqrt{\left\langle N^{2}\right\rangle} \rightarrow 0$.

Given the definition of the spectral average to be used here, we proceed to the case of a regular HM perturbed by conventionally stable wave vectors.

\section{Usual simulations}

In Fig. 3 we start to examine the spatiotemporal dynamics of homogeneous initial conditions perturbed by small inhomogeneous terms that are linearly stable in the conventional sense discussed in the Introduction. Usual simulations are performed where mode coupling is fully dictated by our original set of governing equations [Eqs. (2)-(5)]; we shall refer to these usual type of simulations as fully connected here. In addition to the initial conditions defined by relations (13), again with $\mathcal{A}=(0,0.185)$, we impose a small inhomogeneous perturbation on mode $A_{2}$ whose strength is much smaller than $|\mathcal{A}|, a_{2}(t=0)=0.001 \mathcal{A}$. The curves for $r=1$ in Fig. 3(a) are made for the same parameters and respective varying values of $\delta_{3}$ that correspond to the outer orbits of Figs. 2(a) and 2(b). As for curve $r=0.8$, we use $\delta_{3}=0.1$. We take $k=0.5$ so as to guarantee that we are well outside the linear instability band. The lower curve $\left(r=1, \delta_{3}=0.0001\right)$ reveals that, in terms of energy transfer, nothing is really happening. Energy stays confined within the region of modes with large wave vectors. Figure 3(b) shows what happens in space and time; in that figure we plot the quantity

$$
C=2\left|A_{1}(x, t)\right|^{2}+\left|A_{2}(x, t)\right|^{2}+\left|A_{3}(x, t)\right|^{2},
$$


(a)

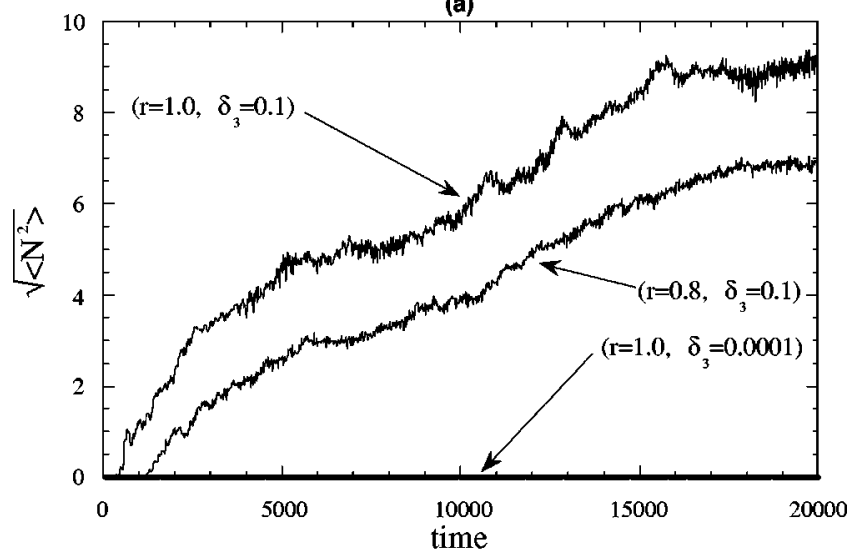

(b)

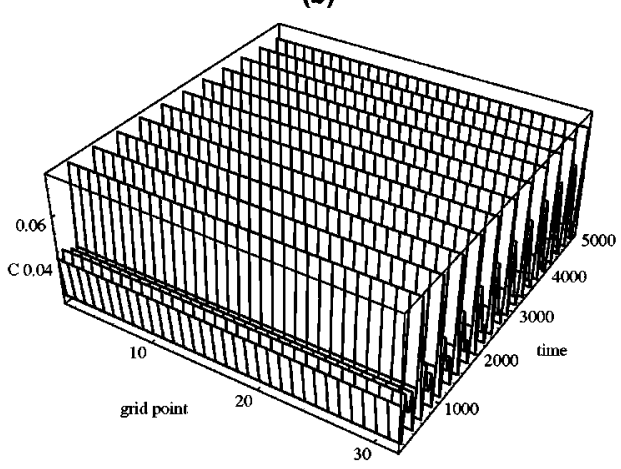

(c)

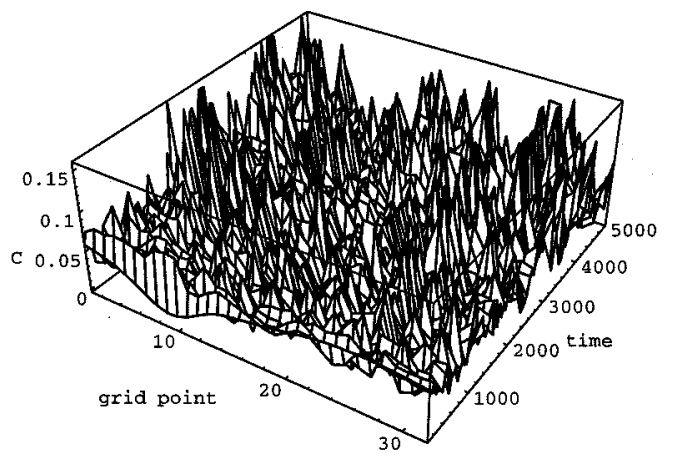

FIG. 3. Conventionally stable HM's: (a) $\sqrt{\left\langle N^{2}\right\rangle}$ vs time, (b) the $C(x, t)$ space-time mesh for $\delta_{3}=0.0001$ and $r=1$, and (c) the $C(x, t)$ space-time mesh for $\delta_{3}=0.1$ and $r=1$. In all cases, $\mathcal{A}$ $=(0,0.185), \quad a_{2}=10^{-3} \mathcal{A}, \quad k=0.5, \quad \mathrm{v}_{g_{1}}=0, \quad \mathrm{v}_{g_{2}}=1, \quad$ and $\quad \mathrm{v}_{g_{3}}$ $=-0.5$. All of the remaining initial conditions are zeroed and $C(x, t)$ is defined in the text.

which is a conserved quantity in the purely homogeneous, uncoupled system, as can be seen from Eqs. (2)-(4). We use $C$ to be free of the fast temporal oscillations generated by the cyclic dynamics on the HM. Figure 3(b) then reveals that, for the small value $\delta_{3}=0.0001$, the dynamics is mostly regular with no energy transfer observed in the full simulations.

As we examine the case $r=1, \delta_{3}=0.1$, where the HM supports chaotic orbits, a striking difference can be appreciated. Here transfer does take place. Energy initially injected at small wave vectors keeps spreading towards fluctuations with progressively smaller length scales. Transfer is arrested, or saturated, only when all modes in the system become involved in the dynamics. This is what is revealed by the

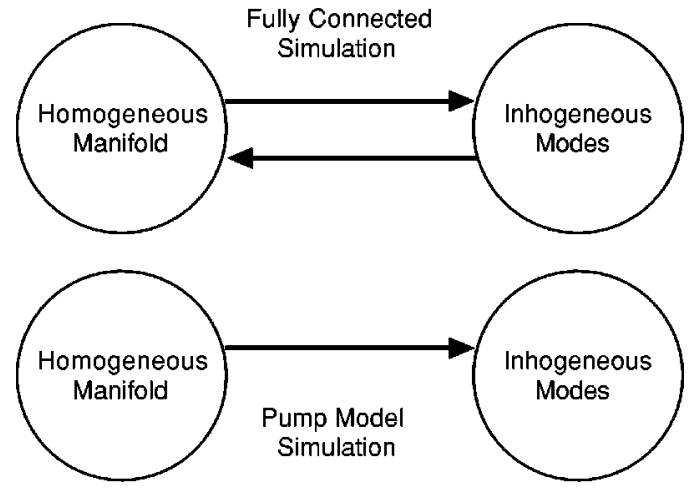

FIG. 4. General schematics of the pump model simulation.

upper curve of Fig. 3(a) and by Fig. 3(c), both cases with $\delta_{3}=0.1$, which is the same value used in Fig. 2(b).

Now, if we keep $\delta_{3}=0.1$ and reduce $r$ to $r=0.8$, the intermediate curve of Fig. 3 allows one to see that energy transfer is inhibited. This is compatible with the fact that the $\mathrm{HM}$ is less chaotic for smaller values of $r$. In the limit $r$ $=0$, in particular, the wave triplets become uncoupled and the dynamics becomes completely integrable with energy transfer no longer occurring.

As mentioned earlier, chaotic low-dimensional subsystems immersed into systems with larger dimensionalities may act much like a thermal source, delivering energy into all the remaining DOF's [18]. This is the basic pattern that we see here.

\section{Pump model simulations}

It is then apparent that we are working with conventionally stable inhomogeneous modes whose dynamics is excited only when the HM supports chaotic orbits. Let us effectively test this idea of a thermal source, or stochastic pump model, as is more usually known. To do that we propose the following numerical procedure. In the simulations we remove all the influence of inhomogeneous modes on the HM, but keep the action of the HM on the inhomogeneous modes. In this way we actually convert the HM into a closed source to which the remaining (inhomogeneous) modes are attached. The general schematics can be found in Fig. 4. If the stochastic pump idea is correct, at least for earlier stages of the dynamics, there must be some agreement between our fully connected simulations and simulations based on the procedure related above. Beyond the point where the fully connected HM loses an appreciable part of its initial energy, the agreement is expected to cease.

We begin with the case of a regular dynamics developing on the HM. Initial conditions are the same as in Fig. 3(b) and the results of our comparisons are shown in Fig. 5(a). One sees that the number of modes remains the same all the way along the simulation both in the fully connected and the pump model approaches. The growth in the number of involved modes is not significant within the time scales used in the computations. On the other hand, as we move to chaotic cases, the dynamics undergoes drastic changes. We see this from Fig. 5(b) where the initial conditions correspond to those of Fig. 3(c). Both in the fully connected and pump simulations, the number of modes grows until saturation. Simulation using the pump model agrees with the fully con- 

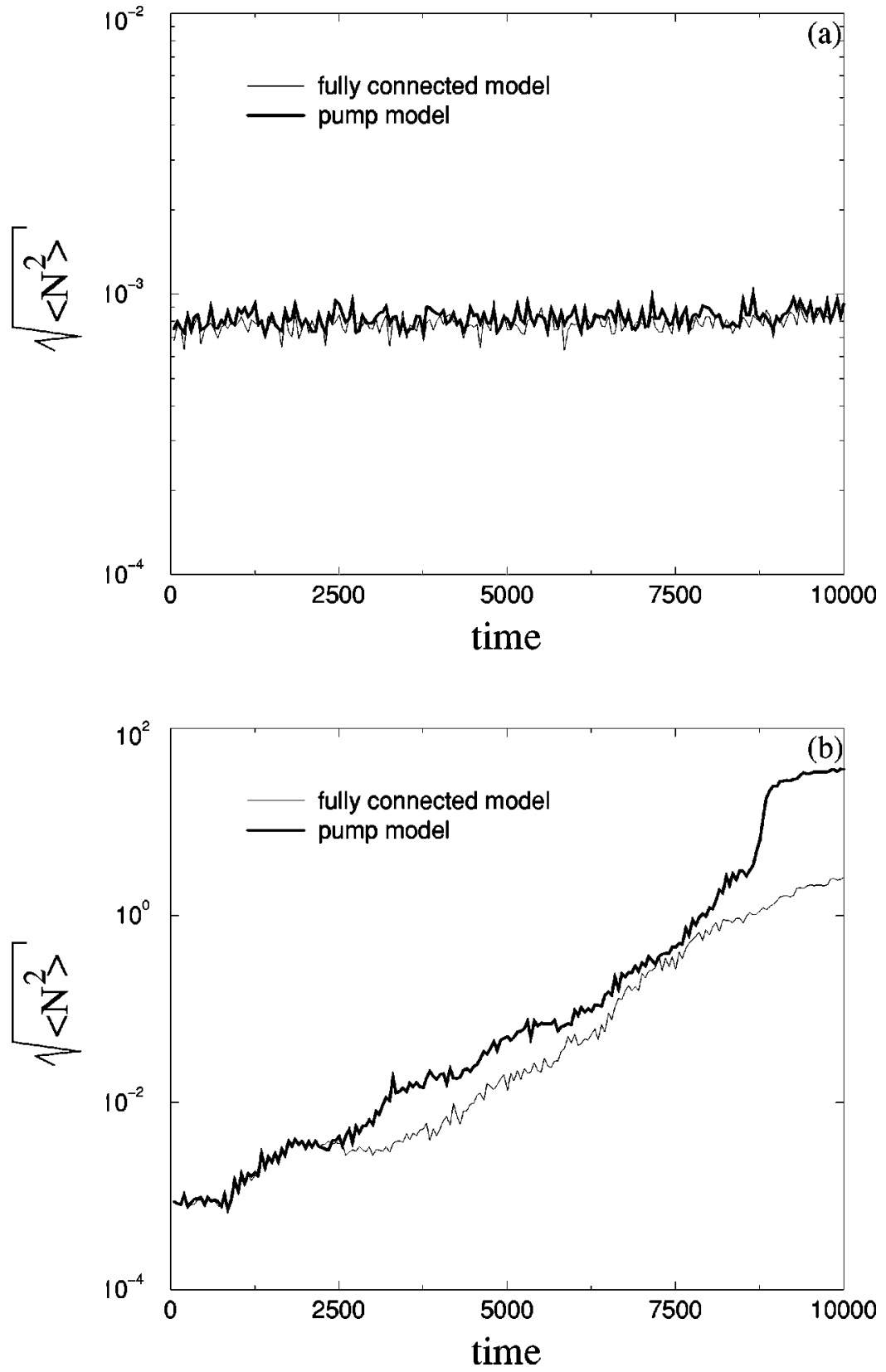

FIG. 5. Comparison of the pump model and fully connected simulations. (a) When diffusion is absent, $\delta_{3}=0.0001$; (b) when diffusion is present, $\delta_{3}=0.1$. In all cases, $\mathcal{A}=(0,0.185), a_{2}$ $=10^{-3} \mathcal{A}, \quad k=0.5, \quad r=1, \quad \mathrm{v}_{g_{1}}=0, \quad \mathrm{v}_{g_{2}}=1$, and $\mathrm{v}_{g_{3}}=-1$. The remaining initial conditions are zeroed. nected one during earlier stages, as anticipated. While the $\mathrm{HM}$ energy is much larger than that contained by the rest of the system, we can actually see the HM as a pump delivering energy to all other modes. However, when the HM energy depletion becomes too large, the simulations begin to move apart from each other. The pump simulations tend to attain saturation earlier because the unceasing energy delivery is much faster there.

\section{UNSTABLE HOMOGENEOUS MANIFOLDS AND SOLITONS}

Our next goal is to complement the previous ideas and look at those situations where the HM is linearly unstable in the conventional sense. In other words, we work with a set of wave vectors that would cause inhomogeneous perturbations to grow, if the HM were static $-k=0.05$. And more than that, we will be working with a set of parameters for which solitons are possible solutions in the uncoupled case, $r=0$; this can lead to localization in our full simulations. We point out that the condition $r=0$ is not sufficient for soliton formation. To allow for solitons in the uncoupled system, one must satisfy the following conditions involving the group velocities, $\mathbf{v}_{g_{2}}<\mathbf{v}_{g_{1}}<\mathbf{v}_{g_{3}}$, as shown in the literature [4]; we take $\delta_{3}=0$ in addition. This is convenient because, under this restriction, the pure HM is completely regular, as said before. So we can focus only on nonintegrable features arising from the existence of inhomogeneous modes in the system. Let us start with the analysis by taking $r=0$. In this limit the triplets are uncoupled and completely integrable, and asymptotic solutions should form regular patterns. That this is so can be observed with the help of Fig. 6. With $r$ $=0$ in Figs. 6(a) and 6(b) one sees a regular pattern of equally interspersed spikes distributed over the space-time plane [Fig. 6(b)]. The regular structure continues to repeat itself indefinitely and no energy transfer takes place [Fig. 6(a)]. 


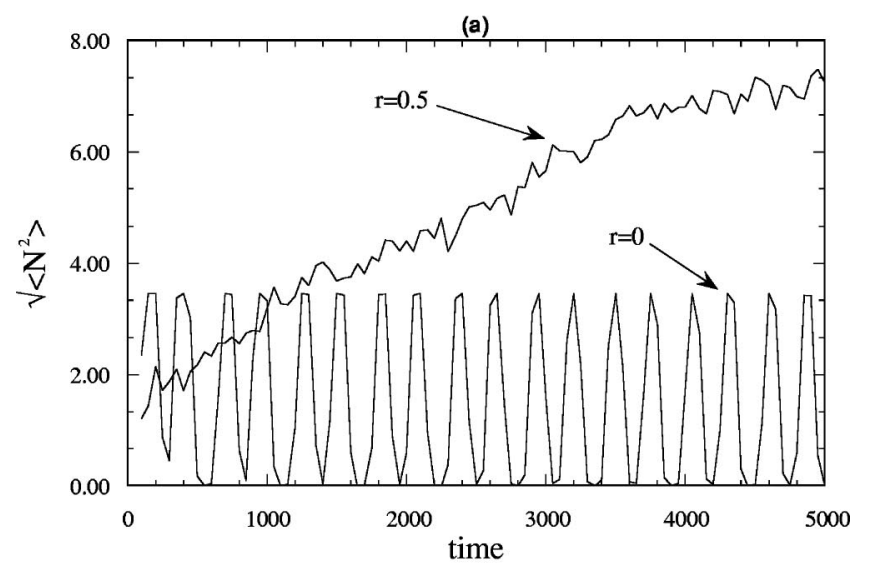

(b)

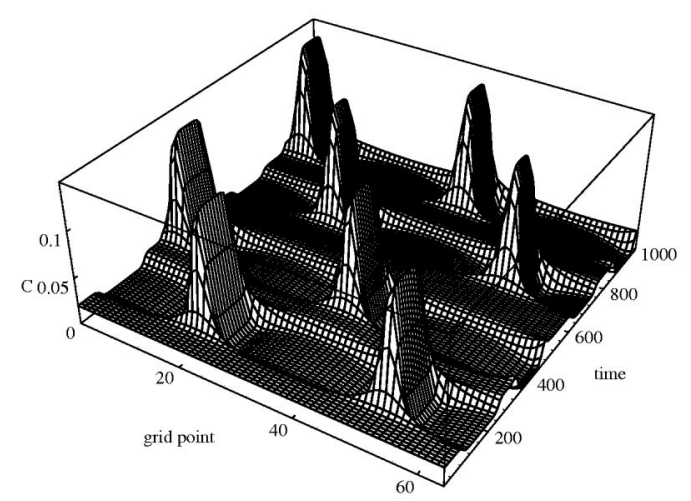

(c)

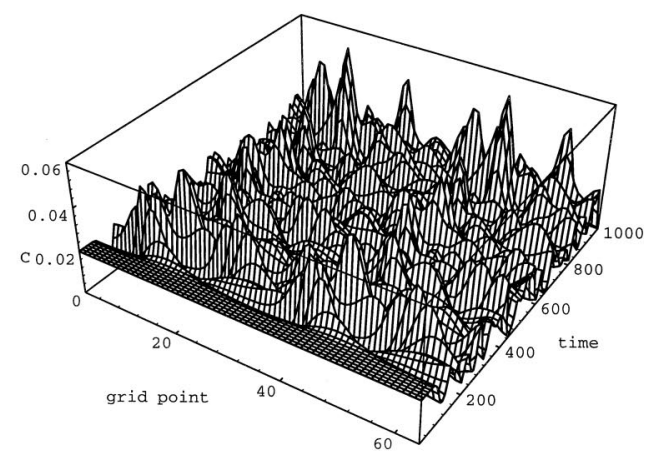

FIG. 6. Conventionally unstable HM's: (a) $\sqrt{\left\langle N^{2}\right\rangle}$ vs time, (b) the $C(x, t)$ space-time mesh for $r=0$, and (c) the $C(x, t)$ space-time mesh for $r=0.5$. In all cases, $\mathcal{A}=(0,0.1), a_{2_{1}}=10^{-3} \mathcal{A}, k=0.05$, and $\delta_{3}=0 . \mathrm{v}_{g_{1}}=0, \mathrm{v}_{g_{2}}=1$, and $\mathrm{v}_{g_{3}}=-1$. The remaining initial conditions are zeroed.

On the other hand, as we turn the coupling factor $r$ on, regularity is destroyed. For $r=0.5$ Fig. 6(c) reveals that spikes can no longer be seen, and Fig. 6(a) that energy transfer now does occur.

In the case of conventional stability one already found out that the drive to spatiotemporal chaos is provided by a possible chaotic dynamics on the HM. Does something similar happen in the present case? To answer the question we first note that the HM by itself is not chaotic here, since we take $\delta_{3}=0$ [19]. Therefore, it cannot provide the drive to spatiotemporal chaos by itself. However, since we now have a second set of active modes, the conventionally unstable modes at the first harmonic, one can wonder if the extended subsystem formed by the HM plus the first harmonics would not be chaotic. To go into this point we simply disconnect the subsystem formed by homogeneous plus first harmonic modes from the rest of the system, and analyze the reduced dynamics. What we find out is pictured in Fig. 7. In the figure we display Poincaré plots where the pair $\operatorname{Re}\left(a_{1_{o}}\right), d \operatorname{Re}\left(a_{1_{o}}\right) / d t$ is recorded each time $\operatorname{Re}\left(a_{1_{1}}\right)$ attains a maximum. From panel (a) it is clear that, for $\delta_{3}=r=0$, the dynamics is periodic and thus integrable. But as we go to larger values of $r$, keeping $\delta_{3}=0$ so as to maintain integrability on the pure HM, what we see is the dynamics of Fig. 7(b). The reduced dynamics is clearly nonperiodic. It can therefore also act like a stochastic source feeding energy into modes with $n \geqslant 2$. Further progress on this topic shall be reported.

\section{CONCLUSIONS}

To begin with, let us recall that we have studied wave systems that, in addition to offering the usual linear stability properties, can also develop a nontrivial dynamics on its homogeneous manifold where all the fields are space independent. Due to this very fact, the effective stability of the purely homogeneous dynamics differs from conventional linear studies that take the homogeneous background as a static entity.

The first conclusion regarding this point comes from the analysis of conventionally stable HM's, where the term conventional actually indicates usual linear calculations considering the HM as a stationary background. Now, a conventionally stable HM can be effectively stable or unstable, depending on the type of dynamics it supports. In the case of a regular dynamics, the HM is effectively stable, but if one goes to the case of a chaotic orbit, effective stability is lost. In this case the HM starts to work like a random or stochastic source, diffusively delivering energy into spatially dependent modes. We conducted a numerical test to verify the reliability of the idea. The test suppresses the influence of inhomogeneous modes on the HM and therefore converts the manifold into a genuine source.

With this stochastic model at hand we conclude that if the $\mathrm{HM}$ is regular, it is not capable of delivering energy into inhomogeneous modes. On the other hand, when chaotic, energy delivery does take place. Full simulations agree with the results of the model.

The other case studied here is that of regular HM's coupled to conventionally unstable inhomogeneous modes. We have focused our study on the range of parameters where the uncoupled triplets may support localized solutions. Starting from homogeneous initial conditions slightly perturbed by inhomogeneities, the uncoupled system evolves towards a regular distribution of space-time spikes. As a result of coupling, energy transfer does occur. In this case, it is also apparent that transfer is at least initially driven by chaos in a subsystem. The subsystem here is not the HM, since we work with a set of parameters for which the HM by itself is totally integrable. In the present case the chaotic subsystem is formed by the HM plus the unstable modes of the first harmonic components. Except for this difference, the stochastic pump model works along similar lines, as previously mentioned. 

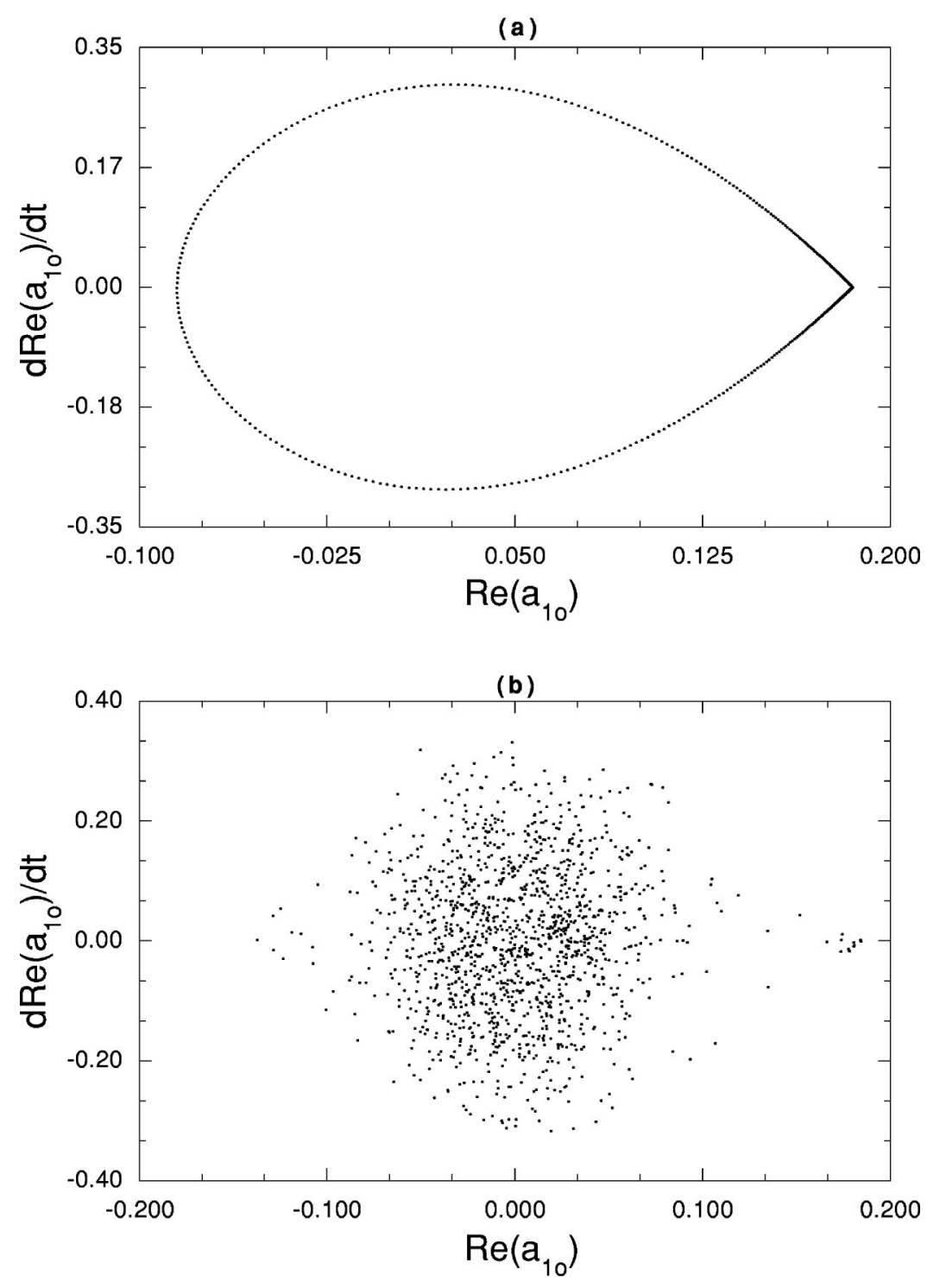

FIG. 7. Poincare plots of the subsystem formed by the HM plus the $n=1$ modes: (a) $r=0$ and (b) 0.5 . The remaining parameters are as in the previous figure.

To summarize our findings, once again we mention that we start the work with the analysis of a chaotic homogeneous manifold displaying conventional linear stability. We indicate that this chaotic subsystem induce energy transfer to modes with progressively smaller wavelengths. We then proceed to a linearly unstable homogeneous manifold that by itself is completely regular. The idea is to show that transfer occurs again and that its drive can be seen again as a chaotic subset of the entire dynamical system.

\section{ACKNOWLEDGMENTS}

This work was partially supported by Financiadora de Estudos e Projetos (FINEP), Conselho Nacional de Desenvolvimento Científico e Tecnológico $(\mathrm{CNPq})$, and Fundação da Universidade Federal do Paraná (FUNPAR), Brazil. Part of the numerical computing was performed on the CRAY computers at the Supercomputing Center of the Universidade Federal do Rio Grande do Sul.
[1] A.C.-L. Chian and F.B. Rizzato, J. Plasma Phys. 51, 61 (1994).

[2] S.R. Lopes and A.C.-L. Chian, Astron. Astrophys. 305, 669 (1996).

[3] A.C.-L. Chian, J.R. Abalde, M.V. Alves, and S.R. Lopes, Sol. Phys. 173, 199 (1997).

[4] D.J. Kaup, A.H. Reiman, and A. Bers, Rev. Mod. Phys. 51, 275 (1979).
[5] D. Walters and G.J. Lewak, J. Plasma Phys. 18, 525 (1977).

[6] R. Pakter, S.R. Lopes, and R. Viana, Physica D 110, 277 (1997).

[7] S.R. Lopes and F.B. Rizzato, Physica D 117, 13 (1998).

[8] A.C.-L. Chian, S.R. Lopes, and J.R. Abalde, Physica D 99, 269 (1996).

[9] R. Sugihara, Phys. Fluids 11, 178 (1968). 
[10] K.S. Karplyuk, V.N. Oraevskii, and V.P. Pavlenko, Plasma Phys. 15, 113 (1973).

[11] V. N. Tsytovich, Nonlinear Effects in Plasma (Plenum, New York, 1970).

[12] W. L. Kruer, The Physics of Laser Plasma Interactions (Addison-Wesley, Redwood City, 1988).

[13] J. de Luca, A. Lichtenberg, and M. Lieberman, Chaos 5, 283 (1995).

[14] F.B. Rizzato, G.I. de Oliveria, and R. Erichsen, Phys. Rev. E 57, 2776 (1998); see references therein about soliton turbulence.
[15] F.B. Rizzato and A.C.-L. Chian, J. Plasma Phys. 48, 71 (1992). [16] R. Pakter, R.S. Schneider, and F.B. Rizzato, Phys. Rev. E 49, 1594 (1994).

[17] R. Erichsen, G.I. de Oliveria, and F.B. Rizzato, Phys. Rev. E 58, 7812 (1998).

[18] A. J. Lichtenberg and M.A. Lieberman, Regular and Chaotic Dynamics (Springer, New York, 1991).

[19] F.J. Romeiras, Phys. Lett. 93A, 227 (1983).

[20] D.U. Martin and H.C. Yuen, Phys. Fluids 23, 1269 (1980).

[21] A. Thyagaraja, Phys. Fluids 24, 1973 (1981). 